\title{
The future of LC-MS for pharmaceutical analysis: an interview with Jun Qu
}

\author{
Jun $\mathrm{Qu}^{*}, 1$ \\ ${ }^{1}$ Department of Pharmaceutical Sciences, School of Pharmacy, University at Buffalo, New York, NY, USA \\ * Author for correspondence: Tel.: +1 716213 8489; junqu@buffalo.edu
}

\begin{abstract}
Jun Qu speaks to Sankeetha Nadarajah, Editor of Bioanalysis: Jun Qu is the group leader of the proteomics and pharmaceutical analysis lab of SUNY-Buffalo (NY, USA) and a Professor in the Department of Pharmaceutical Sciences. His research is focused on the study of Clinical and Pharmaceutical Proteomics and Pharmaceutical Analysis using LC-MS-based strategies. His research programs include high-resolution and large-scale expression profiling of pathological proteomes, for the discovery of novel disease/therapeutics biomarkers using gel-free proteomic methods; sensitive identification, localization and quantification of post-translational modifications in tissue proteomes such as these in myocardium, using novel anti-PTM affinity capture and alternating Collision-induced dissociation/Electron transfer dissociation to obtain abundant PTM information; targeted investigation of marker proteins that are of high interests for clinical and pharmaceutical study, using highly sensitive nano-LC/SRM-based methods; and highly sensitive and accurate investigation of the PK of biotherapeutics using LC-MS.
\end{abstract}

First draft submitted: 15 November 2017; Published online: 16 February 2018

Keywords: bioanalysis $\bullet$ LC-MS; biotherapeutics $\bullet$ pharmaceutical analysis $\bullet$ proteomics

\section{What is your current research focus?}

Currently, I am working in LC-MS-based bioanalysis, mainly in the protein area. So mainly my research actually encumbers two related but quite different areas. The first area is global proteomics; we developed a number of label-free proteomic methods, to enable large-scale bioanalysis on the global proteomics level - for example for the quantification of thousands and thousands of proteins at a large scale in hundreds of samples. Another research area, which is more focused, is the bioanalysis of protein drug biotherapeutics, most of which are antibody based.

In recent years, our lab has developed a number of technologies to address the challenges in the bioanalysis of antibody drugs. To address these we developed a Trapping-Micro-LC-MS technology, which has a high sensitivity similar to nano-LC-MS, but has a high throughput and robustness like traditional high flow LC-MS. Therefore, the method is able to achieve a low nanogram per mill sensitivity, but also has a very high throughput (several minutes per sample), and can run thousands of samples without having to stop. Therefore, we think it can be a practical technology for both the pharmaceutical industry and for large scale analysis.

Another technique, we have developed, can be used for tissue analysis. For tissue analysis you need to do two things right. Firstly, you need to get rid of the blood, and secondly you need to extract the target efficiently and reproducibly from the tissue. In order words, to address this, we developed a differential labeling method to get the perfect perfusion of the sample. We also achieved efficient and reproducible extraction of the drug, and the receptor or biomarker (for example), from the tissue. Additionally, another technology, we developed, is for the accurate analysis, to improve the accuracy, and we call this the hybrid calibration method.

\section{What are the challenges of implementing LC-MS for biotherapeutic quantification?}

For the challenges, there are multiple dimensions. Right now, although LC-MS looks like it is a very promising alternative to ligand binding, and other methods used for protein drug analysis, the reality is that it is very challenging. First off is the sensitivity; we always think LC-MS is a very sensitive method for bioanalysis, but this is not the case for protein analysis for a number of reasons. First, the protein drug is a big molecule, which puts a disadvantage on more independent methods like LC-MS. Second, it is very important to dilute the biological sample; so for small molecule analysis, we usually concentrate the sample, but for protein analysis we do this the 
opposite way, which decreases the sensitivity. And most importantly for protein analysis, the $\mathrm{S} / \mathrm{N}$ are usually very low because the chemical noise is very high.

\section{What are some of the key issues of biotherapeutic quantification in tissues using LC-MS?}

There are multiple challenges for tissue analysis. Right now the first thing I would mention is that for tissue analysis it is very important, and getting more and more important because of the coming up of new constructs in biotherapeutics (e.g., bispecific antibodies, ADCs, etc.), that we figure out how much of the drug is actually in the tissue. Also, in the tissue we need to analyze the target, because for most of the constructs and the tissue target, not only the drug but also the target needs to be analyzed. Therefore, the first challenge is to put tissue sample manually, so how to extract the drug out the tissue efficiently and reproducibility is the first challenge. And the second challenge is the sensitivity, because the tissue levels are usually 10- or 100-times lower than circulating levels, which means we need an ultrasensitive method to overcome this. And yet another issue is accuracy, so we really need to get a good accuracy for tissue analysis, however this can be very difficult to evaluate.

\section{Where do you think this current trend for LC-MS quantification is headed?}

In the near future, I think LC-MS will be more popular in the field of antibody drug analysis for a number of reasons. First, for LC-MS, the method development is cheaper and quicker, so usually a method can be developed in a week as opposed to several months by ELISA and other traditional methods. Second, LC-MS can work in different matrices - it can work in plasma and tissue equally well - and also the LC-MS can quantify not only the drug but also the receptors in the tissue, which is very difficult to do using other methods. So as of recently, when the biospecific antibodies and the ADCs came out, it is very important to do tissue analysis, and LC-MS is by far the best method for this. People need to figure out how much the target is still in the tissue during the course of treatment, and also need to figure out the biomarkers for drug effect and side effect, and LC-MS has multiplexing ability to analyze everything in just one analysis. So, I am very optimistic with the LC-MS technology in the near future, in the pharmaceutics area, and in both industry and academic.

Disclaimer

The opinions expressed in this interview are those of the interviewee and do not necessarily reflect the views of Future Science Ltd.

Financial \& competing interests disclosure

The author has no relevant affiliations or financial involvement with any organization or entity with a financial interest in or financial conflict with the subject matter or materials discussed in the manuscript. This includes employment, consultancies, honoraria, stock ownership or options, expert testimony, grants or patents received or pending, or royalties.

No writing assistance was utilized in the production of this manuscript. 